\title{
Provably $\Delta_{2}^{0}$ and weakly descending chains *
}

\author{
Toshiyasu Arai \\ Graduate School of Science, Chiba University \\ 1-33, Yayoi-cho, Inage-ku, Chiba, 263-8522, JAPAN \\ tosarai@faculty.chiba-u.jp
}

\begin{abstract}
In this note we show that a set is provably $\Delta_{2}^{0}$ in the fragment $I \Sigma_{n}$ of arithmetic iff it is $I \Sigma_{n}$-provably in the class $D_{\alpha}$ of $\alpha$-r.e. sets in the Ershov hierarchy for an $\alpha<_{\varepsilon_{0}} \omega_{1+n}$, where $<_{\varepsilon_{0}}$ denotes a standard $\varepsilon_{0}$-ordering.

In the Appendix it is shown that a limit existence rule $(\operatorname{LimR})$ due to Beklemishev and Visser becomes stronger when the number of nested applications of the inference rule grows.
\end{abstract}

\section{Introduction}

Thoroughout this paper, we identify a predicate $A$ with its characteristic function

$$
A\left(x_{1}, \ldots, x_{n}\right)= \begin{cases}0 & \text { if } A\left(x_{1}, \ldots, x_{n}\right) \\ 1 & \text { otherwise }\end{cases}
$$

Natural numbers $c$ are identified with the sets $\{n \in \mathbb{N}: n<c\}$.

The following Limit Lemma due to Shoenfield is a classic in computability theory.

Theorem 1 (Limit Lemma)

$A$ set $A$ of natural numbers is $\Delta_{2}^{0}$ iff there is a binary (primitive) recursive predicate $f: \omega \times \omega \rightarrow 2=\{0,1\}$ such that

$$
\forall c\left[\lim _{w \rightarrow \infty} f(c, w)=A(c)\right] .
$$

Moreover the theorem is provable uniformly in $B \Sigma_{1}^{0} \subseteq I \Sigma_{1}^{0}$, cf. [4, pp. 89-91. Let us call the predicate $f$ a witnessing predicate for $A \in \Delta_{2}^{0}$.

In this paper we address a problem asking what can we say about the rate of convergences of the predicate $f$ under the assumption that the set $A$ is provably $\Delta_{2}^{0}$ in a formal (sound) theory $\mathrm{T}$ ?

*Dedicated to the occasion of Chong Chi Tat's 60th birthday 
This is a problem on a hierarchy. The class of $\Delta_{2}^{0}$-sets is classified in the Ershov hierarchy, 3]. A recent article [1] due to F. Stephan, Y. Yang and L. $\mathrm{Yu}$ is a readable contribution to the hierarchy, to which we refer as a standard text.

The $\alpha$-th level of the Ershov hierarchy is denoted $D_{\alpha}$ for notations $\alpha$ of constructive ordinals, and a set in $D_{\alpha}$ is said to be an $\alpha$-r.e. set.

It is known, as usual in hierarchic problems indexed by constructive ordinals, that $D_{\alpha}$ depends heavily on notations $\alpha$, i.e., the order type of $\alpha$ does not determine the set $D_{\alpha}$. By reason of this dependency let us fix a standard elementary recursive well ordering $<_{\alpha}$ of type $\alpha$. I don't want to discuss here what is a 'standard ordering' or a 'natural well ordering'. We assume that $\mathrm{EA}=\mathrm{I} \Delta_{0}^{0}+\exp$, Elementary Recursive Arithmetic, proves some algebraic facts on the ordering $<_{\alpha}$. For the case $\alpha=\varepsilon_{0}$, what we need on $<_{\varepsilon_{0}}$ can be found in, e.g., [10].

In what follows let us drop the subscript $\alpha$ in $<_{\alpha}$ when no confusion likely occurs.

Definition 2 (Stephan-Yang-Yu [1])

Let $K \in \operatorname{dom}(<)$, the domain of the order $<$.

A set $A$ of natural numbers is $K$-r.e. with respect to $<$ iff there exist a binary recursive predicate $f$, and a recursive function $h: \omega \times \omega \rightarrow K=\{\beta \in$ $\operatorname{dom}(<): \beta<K\}$ such that

1.

$$
\text { (weakly descending) } K>h(c, w) \geq h(c, w+1)
$$

2.

$$
\text { (lowering) } f(c, w) \neq f(c, w+1) \rightarrow h(c, w)>h(c, w+1)
$$

3.

$$
\forall c\left[\lim _{w \rightarrow \infty} f(c, w)=A(c)\right]
$$

Roughly speaking, a set is $K$-r.e. if the convergence of its witnessing predicate follows from the fact that weakly decreasing functions in $K$ have to be constant eventually.

Now suppose that we have a proof-theoretic analysis of a formal (and sound) theory T, e.g., a cut-elimination through a transfinite induction along a standard well ordering $<$. It, then, turns out that $A$ is provably $\Delta_{2}^{0}$ in $\mathrm{T}$ iff $\mathrm{T}$ proves the fact that $A \in D_{K}$ with respect to $<$ for a $K \in \operatorname{dom}(<)$.

Though, in this paper, we restrict our attention to $\mathrm{T}=I \Sigma_{n}^{0}$ of fragments of first order arithmetic as a concrete example, where the order $<$ denotes a standard well ordering of type $\varepsilon_{0}$, it is easy to see that our proof works also for stronger theories, e.g., second order arithmetic $\Pi_{1}^{1}-\mathrm{CA}_{0}$ and fragments of set theories.

In Section 2 it is shown that for each $n \geq 1$, a set is provably $\Delta_{2}^{0}$ in the fragment $I \Sigma_{n}$ iff it is $I \Sigma_{n}$-provably in the class $D_{\alpha}$ for an $\alpha<_{\varepsilon_{0}} \omega_{1+n}$ (Theorem 4). 
Also any provably $\Sigma_{2}^{0}$-function has a Skolem function $F(c)=\lim _{w \rightarrow \infty} f(c, w)$ as limits of an $f$, whose convergence is ensured by weakly descending chains of ordinals (Theorem 9). Moreover the 2-consistency $\operatorname{RFN}_{\Pi_{3}^{0}}\left(I \Sigma_{n}^{0}\right)$ is seen to be equivalent over Primitive Recursive Arithmetic PRA to the fact that every primitive recursive weakly descending chain of ordinals $<\omega_{1+n}$ has a limit(Theorem 101).

In Section 3 it is shown that a set is provably $\Delta_{2}^{0}$ in Elementary Recursive Arithmetic EA iff it is EA-provably in the class $D_{n}$ of a finite level (Theorem 11). Our proof seems to be a neat application of the Herbrand's theorem.

The Appendix $\mathrm{A}$ contains another application of Herbrand's theorem. We consider, over EA, an inference rule $(\operatorname{Lim} R)$ in [2, which concludes the convergence of an elementary recursive series $\{h(n)\}_{n}$ under the assumption that the series is weakly decreasing almost all $n$. Note that $(\mathrm{LimR})$ is an inference rule, and not an axiom(sentence).

On the other side, let $L \Sigma_{1}^{-(k)}$ denote the schema in [5], saying that any nonempty $\Sigma_{1}^{0} k$-ary predicate has the least tuple, which is least with respect to the lexicographic ordering on $\mathbb{N}^{k}$.

It is shown that $L \Sigma_{1}^{-(k)}$ is equivalent to the $k$-nested applications of ( $\mathrm{LimR}$ ). In [5], Corollary 2.11 it was shown that $\left\{L \Sigma_{1}^{-(k)}\right\}_{k}$ forms a proper hierarchy, i.e., $L \Sigma_{1}^{-(k+1)} \vdash \operatorname{Con}\left(L \Sigma_{1}^{-(k)}\right)$. Hence we conclude that a $(k+1)$-nested application of $(\mathrm{LimR})$ proves the consistency of the $k$-nested applications of $(\mathrm{LimR})$.

\section{Provably $\Delta_{2}^{0}$ in $I \Sigma_{n}^{0}$}

Let LEA [EA] denote the Lower Elementary Recursive Arithmetic [Elementary Recursive Arithmetic], which is a first-order theory in the language having function constants for each code(algorithm) of lower elementary recursive function [function constants for each code of elementary recursive function], resp. Cf. [8] and [9] for these classes of subrecursive functions. Induction schema is restricted to quantifier-free formulas in the language. The axioms of the theories LEA, EA are purely universal ones.

Let $I \Sigma_{n}^{0}$ denote the fragment of arithmetic, which is a first-order theory in the language of LEA, and Induction schema is restricted to $\Sigma_{n}^{0}$ formulas. Here a $\Sigma_{0}^{0}$ formula is a quantifier-free formula. $I \Sigma_{0}^{0}$ is another name for LEA.

Let $<_{\varepsilon_{0}}$ denote a standard $\varepsilon_{0}$-ordering. We assume that EA proves some algebraic facts on the ordering $<_{\varepsilon_{0}}$. What we need on $<_{\varepsilon_{0}}$ can be found in, e.g., 10 .

In what follows let us drop the subscript $\varepsilon_{0}$ in $<_{\varepsilon_{0}}$ when no confusion likely occurs.

For a class $\Phi$ of formulas and an ordinal $\alpha$ let $T I(\Phi, \alpha)$ denote the schema of transfinite induction up to $\alpha$ and applied to a formula $\varphi \in \Phi$ :

$$
\forall \beta[\forall \gamma<\beta \varphi(\gamma) \rightarrow \varphi(\beta)] \rightarrow \forall \beta<\alpha \varphi(\beta) .
$$

Let

$$
\omega_{0}:=1, \omega_{1+n}:=\omega^{\omega_{n}} .
$$


Here is a folklore result on provability of the restricted transfinite induction schemata in fragments of arithmetic.

Theorem 3 (See, e.g., [10])

For each $n \geq 0, I \Sigma_{n}^{0} \vdash T I\left(\Pi_{1}^{0}, \alpha\right)$ iff $\alpha<\omega_{1+n}$.

The following Theorem 4 states that for positive integers $n$, a set is provably $\Delta_{2}^{0}$ in $I \Sigma_{n}^{0}$ iff it is $I \Sigma_{n}^{0}$-provably in the class $D_{\alpha}$ of $\alpha$-r.e. sets in the Ershov hierarchy for an $\alpha<_{\varepsilon_{0}} \omega_{1+n}$. Moreover (weakly descending) and (lowering) are provable in EA.

Theorem 4 For positive integers $n$, the following are equivalent for quantifierfree $A, B$ and a free variable $c$.

1. $I \Sigma_{n}^{0}$ proves

$$
\forall x \exists y A(x, y, c) \leftrightarrow \exists z \forall u B(z, u, c)
$$

2. There exists a binary elementary recursive predicate $f$, an ordinal $K<$ $\omega_{1+n}$ and an elementary recursive function $h: \omega \times \omega \rightarrow K$ such that

(a) (weakly descending)

$$
\mathrm{EA} \vdash K>h(c, w) \geq h(c, w+1)
$$

(b) (lowering)

$$
\mathrm{EA} \vdash f(c, w) \neq f(c, w+1) \rightarrow h(c, w)>h(c, w+1)
$$

(c)

$$
\begin{aligned}
\text { EA } & \vdash \\
\text { EA } & \vdash \lim _{w \rightarrow \infty} f(c, w)=0 \rightarrow \exists z \forall u B(z, u, c) \\
I \Sigma_{n}^{0} & \vdash \quad \exists z \forall u B(z, u, c) \rightarrow \forall x \exists y A(x, y, c)
\end{aligned}
$$

where the ordering $<$ denotes a standard $\varepsilon_{0}$-ordering $<_{\varepsilon_{0}}$.

First note that by Theorem 3 we have $\Sigma_{1}^{0}$-minimization up to each ordinal less than $\omega_{1+n}$ in $I \Sigma_{n}^{0}$. Hence $\exists \alpha<_{\varepsilon_{0}} K\left[\alpha=\min _{<_{\varepsilon_{0}}}\{\beta: \exists w[\beta=h(c, w)]\}\right]$. Pick a $w$ so that the least $\alpha=h(c, w)$. Assuming that EA (a fortiori $I \Sigma_{n}^{0}$ ) proves (weakly descending) and (lowering), we have

$$
I \Sigma_{n}^{0} \vdash \forall u \geq w[f(c, u)=f(c, w)] .
$$

Therefore the convergence of the predicate $f$ is shown in $I \Sigma_{n}^{0}$. Also

$$
I \Sigma_{n}^{0} \vdash \forall x \exists y A(x, y, c) \leftrightarrow \lim _{w \rightarrow \infty} f(c, w)=0 .
$$

The converse follows from the following Reduction Theorem 5 
The theorem says that if a disjunction $\exists x \forall y \neg A(x, y, c) \vee \exists z \forall u B(z, u, c)$ of $\Sigma_{2}^{0}$-formulas is provable in $I \Sigma_{n}^{0}$, then one can construct an elementary recursive predicate $f$ whose limit tells us which disjunct is true. The convergence of $f$ is ensured by a descending function $h$ in ordinals $<\omega_{1+n}$. Moreover these are all provable in EA.

Assuming the convergence of $f$ (, which is provable in $I \Sigma_{n}^{0}$ ) this is a provable version of the classical Reduction Property of $\Sigma_{2}^{0}$ sets to $\Delta_{2}^{0}$ sets. The point is that the $\Delta_{2}^{0}$ sets $\left\{c: \lim _{w \rightarrow \infty} f(c, w)=0\right\}$ are in a level $D_{<\omega_{1+n}}$ of Ershov hierarchy, demonstrably in $I \Sigma_{n}^{0}$.

Theorem 5 (Reduction Property) Let $n \geq 1$.

Suppose $I \Sigma_{n}^{0} \vdash \exists x \forall y \neg A(x, y, c) \vee \exists z \forall u B(z, u, c)$ for quantifier-free $A, B$. Then there exists an elementary recursive predicate $f$, an ordinal $K<\omega_{1+n}$ and an elementary recursive function $h$ such that

1. (weakly descending)

$$
\mathrm{EA} \vdash K>h(c, w) \geq h(c, w+1)
$$

2. (lowering)

$$
\mathrm{EA} \vdash f(c, w) \neq f(c, w+1) \rightarrow h(c, w)>h(c, w+1)
$$

3. (reduction)

$$
\begin{aligned}
& \text { EA } \vdash \lim _{w \rightarrow \infty} f(c, w)=0 \rightarrow \exists z \forall u B(z, u, c) \\
& \text { EA } \vdash \lim _{w \rightarrow \infty} f(c, w)=1 \rightarrow \exists x \forall y \neg A(x, y, c)
\end{aligned}
$$

In what follows, given a $I \Sigma_{n}^{0}$-proof of $\exists x \forall y \neg A(x, y, c) \vee \exists z \forall u B(z, u, c)$ let us construct a predicate $f$, an ordinal $K<\omega_{1+n}$ and a function $h$ enjoying (weakly descending), (lowering) and (reduction).

Let $p(x, y, c)$ denote the characteristic function of the predicate

$$
A\left((x)_{0},(y)_{0}, c\right) \rightarrow B\left((x)_{1},(y)_{1}, c\right),
$$

where $(x)_{i}(i=0,1)$ denotes the projections of the pairing function.

Then

$$
\exists x \forall y[p(x, y, c)=0]
$$

is provable in $I \Sigma_{n}^{0}$.

\subsection{Infinitary derivations}

In what follows let us consider (finite or infinite) derivations in one-sided sequent calculi. Given a finite derivation of $\exists x \forall y[p(x, y, c)=0]$ in $I \Sigma_{n}^{0}$, first eliminate cut inferences partially to get a derivation of the same formula in which any cut formula is $\Sigma_{n}^{0}$. 
Next embed the derivation into an infinite derivation of the sentence

$$
\exists x \forall y[p(x, y, \bar{c})=0]
$$

with the $c$-th numeral $\bar{c}$. Then eliminate cut inferences to get a cut-free derivation $P_{c}$ of the same sentence. As usual the depth of $P_{c}$ is bounded by an ordinal $K<\omega_{1+n}$ uniformly, i.e., $\forall c\left[\operatorname{dp}\left(P_{c}\right)<K\right]$.

In the derivation $P_{c}$, the initial sequents are

$$
\text { (Int) } \Gamma, E
$$

for true equation $E$. The equation $E$ is called the main formula of the initial sequent.

In what follows we identify the closed term $t$ with the numeral $\bar{n}$ of its value $n=\operatorname{val}(t)$.

Note that the value of closed terms and truth values of equations in LEA are elementary recursively computable. The initial sequents are regarded as inference rules with empty premiss (upper sequent), and with the empty list of side formulas.

The inference rules are $(\exists),(\forall)$, and the repetition rule $($ Rep $)$. These are standard ones.

$$
\frac{\Gamma, B(\bar{n})}{\Gamma, \exists x B(x)}(\exists) ; \frac{\cdots \quad \Gamma, B(\bar{n}) \cdots(n \in \omega)}{\Gamma, \forall x B(x)}(\forall) ; \frac{\Gamma}{\Gamma}(\operatorname{Rep})
$$

where $\exists x B(x)$ in the $(\exists)$ and $\forall x B(x)$ in the $(\forall)$ are the main formula of the inference, and $B(\bar{n})$ are side formulas of the inferences. The inference (Rep) has no main nor side formulas. [6].

Our infinitary derivations are equipped with additional informations as in

Definition 6 An infinitary derivation is a sextuple

$$
D=(T, \text { Seq }, \text { Rule, } M f m l, S f m l, \text { ord })
$$

which enjoys the following conditions. The naked tree of $D$ is denoted $T=$ $T(D)$.

1. $T \subseteq{ }^{<\omega} \omega$ is a tree with its root $\emptyset$ such that

$$
a *\langle n\rangle \in T \& m<n \Rightarrow a *\langle m\rangle \in T .
$$

2. $\operatorname{Seq}(a)$ for $a \in T$ denotes the sequent situated at the node $a$.

If $\operatorname{Seq}(a)$ is a sequent $\Gamma$, then it is denoted

$$
a: \Gamma \text {. }
$$

3. Rule $(a)$ for $a \in T$ denotes the name of the inference rule with its lower sequent $\operatorname{Seq}(a)$. 
4. $M f m l(a)$ for $a \in T$ denotes the main formula of the inference rule Rule $(a)$. When Rule $(a)=(\operatorname{Rep})$, then $\operatorname{Mfml}(a)=\emptyset$.

5. $\operatorname{Sfml}(a *\langle n\rangle)$ for $a *\langle n\rangle \in T$ denotes the side formula of the inference rule $\operatorname{Rule}(a)$, which is in the $n$-th upper sequent, i.e., $\operatorname{Sfml}(a *\langle n\rangle) \in$ $\operatorname{Seq}(a *\langle n\rangle)$. When Rule $(a)=(\operatorname{Rep}),(\operatorname{Int})$, then $\operatorname{Sfml}(a *\langle n\rangle)=\emptyset$.

6. $\operatorname{ord}(a)$ for $a \in T$ denotes the ordinal $<_{\varepsilon_{0}} K$ attached to $a$.

7. The sextuple ( $T, S e q$, Rule, $M f m l, S f m l$,ord $)$ has to be locally correct with respect to inference rules of the infinitary calculus and for being well founded tree $T$.

In a derivation each inference rule except (Int) receives the following nodes:

$\frac{a *\langle 0\rangle: \Gamma, B(\bar{n})}{a: \Gamma, \exists x B(x)}(\exists) ; \frac{\cdots \quad a *\langle n\rangle: \Gamma, B(\bar{n}) \quad \cdots(n \in \omega)}{a: \Gamma, \forall x B(x)}(\forall) ; \frac{a *\langle 0\rangle: \Gamma}{a: \Gamma}(\operatorname{Rep})$

The ordinals $\operatorname{ord}_{c}(a)$ in the inference $(\forall)$

$$
\frac{\cdots \quad a *\langle n\rangle: \Gamma, B(\bar{n}) \quad \cdots(n \in \omega)}{a: \Gamma, \forall x B(x)}(\forall)
$$

enjoys

$$
\operatorname{ord}_{c}(a)>\operatorname{ord}_{c}(a *\langle n\rangle)=\operatorname{ord}_{c}(a *\langle m\rangle)
$$

for any $n, m$.

As in [6] we see that the function $c \mapsto P_{c}$ is elementary recursive. We denote $P_{c}=\left(T_{c}\right.$, Seq $_{c}$, Rule $_{c}, M f m l_{c}, S f m l_{c}$, ord $\left._{c}\right)$.

\subsection{Searching witnesses of $\Sigma_{2}^{0}$ in derivations}

Let us define a tracing function $\sigma(c, i) \in T_{c}=T\left(P_{c}\right)$.

The function $\{\sigma(c, w)\}_{w}$ indicates the trail in the proof tree $T_{c}$ in which we go through in searching a witness $x_{a}$ of $\exists x \forall y[p(x, y, \bar{c})=0]$, and verifying $\forall y\left[p\left(x_{a}, y, \bar{c}\right)=0\right]$.

1. $\sigma(c, 0)=\emptyset($ root $)$.

In what follows let $a=\sigma(c, w)$.

2. Until $\operatorname{Seq}_{c}(a)$ is an upper sequent of an $(\forall)$, go to the leftmost branch:

$$
\sigma(c, w+1)=a *\langle 0\rangle .
$$

For example

$$
\frac{a *\langle 0\rangle: \Gamma, \exists x \forall y[p(x, y, \bar{c})=0], \forall y\left[p\left(x_{a}, y, \bar{c}\right)=0\right]}{a: \Gamma, \exists x \forall y[p(x, y, \bar{c})=0]}
$$


3. The case when $\operatorname{Rule}_{c}(b)=(\forall)$ with $a=b *\langle n\rangle$. Namely $\operatorname{Seq}_{c}(a)$ is the $n$-th upper sequent of an $(\forall)$.

$$
\frac{\cdots \quad a: \Gamma, p\left(x_{a}, y_{a}, \bar{c}\right)=0 \quad \cdots}{\Gamma, \forall y\left[p\left(x_{a}, y, \bar{c}\right)=0\right]}(\forall)
$$

$x_{a}, y_{a}$ are closed terms.

(a) If $p\left(x_{a}, y_{a}, \bar{c}\right)=0$ is a TRUE equation, $\sigma(c, w+1)=a \oplus 1$, the next right to the $a$ :

$$
\frac{\sigma(c, w): \Gamma, p\left(x_{a}, y_{a}, \bar{c}\right)=0 \quad \sigma(c, w+1): \Gamma, p\left(x_{a}, y_{a}+1, \bar{c}\right)=0}{\Gamma, \forall y\left[p\left(x_{a}, y, \bar{c}\right)=0\right]}(\forall)
$$

where for an $a=\left(a_{0}, \ldots, a_{n-2}, a_{n-1}\right) \in{ }^{<\omega} \omega$

$$
a \oplus 1=\left(a_{0}, \ldots, a_{n-2}, a_{n-1}+1\right)
$$

if $\operatorname{lh}(a)=n>0$.

$\emptyset \oplus 1$ is defined to be $\emptyset$.

(b) Otherwise $\sigma(c, w+1)=a *\langle 0\rangle$, i.e., go to the leftmost branch from $a$.

$$
\frac{\frac{\sigma(c, w+1): \Delta \quad \cdots}{\sigma(c, w): \Gamma, p\left(x_{a}, y_{a}, \bar{c}\right)=0} \quad \cdots}{\Gamma, \forall y\left[p\left(x_{a}, y, \bar{c}\right)=0\right]}(\forall)
$$

It is easy to see that the function $(c, w) \mapsto \sigma(c, w)$ is elementary recursive since $\max \left(\left\{(\sigma(c, w))_{i}: i<\operatorname{lh}(\sigma(c, w))\right\} \cup\{\operatorname{lh}(\sigma(c, w))\}\right) \leq w$.

Once $\sigma(c, w)$ is on an $(\forall)$, the tracing function goes through the upper sequents as long as the equations $p\left(x_{a}, y_{a}, \bar{c}\right)=0$ is TRUE.

It is intuitively clear that after a finite number of steps, the sequence $\{\sigma(c, w)\}_{w}$ goes through the upper sequents of an $(\forall)$ :

$$
\frac{\sigma\left(c, w_{0}\right): \Gamma, p\left(x_{a}, 0, \bar{c}\right)=0 \quad \cdots \quad \sigma\left(c, w_{0}+y\right): \Gamma, p\left(x_{a}, \bar{y}, \bar{c}\right)=0 \quad \cdots}{\Gamma, \forall y\left[p\left(x_{a}, y, \bar{c}\right)=0\right]}
$$

since $\forall y\left[p\left(x_{a}, y, \bar{c}\right)=0\right]$ is true for an $x_{a}$. We will know at the limit the fact, i.e., for $x=\left(x_{a}\right)_{0}$ and $z=\left(x_{a}\right)_{1}$

$$
\exists y A(\bar{x}, y, \bar{c}) \rightarrow \forall u B(\bar{z}, u, \bar{c})
$$

is true.

Now let us define an elementary recursive predicate $f$ as follows.

1. $f(c, 0)=1$. 
2. Alternate values $f(c, w+1)=1-f(c, w)$ if $\operatorname{Seq}_{c}(\sigma(c, w+1))$ is an upper sequent of an inference other than $(\forall)$.

3. Suppose $\operatorname{Seq}_{c}(\sigma(c, w+1))$ is the $n$-th upper sequent of an $(\forall)$, and $\sigma(c, w+$ 1) $=b *\langle n\rangle$.

$$
\frac{\cdots \quad b *\langle n\rangle: \Gamma, A\left(x_{b},(\bar{n})_{0}, \bar{c}\right) \rightarrow B\left(z_{b},(\bar{n})_{1}, \bar{c}\right) \quad \cdots}{b: \Gamma, \forall y, u\left[A\left(x_{b}, y, \bar{c}\right) \rightarrow B\left(z_{b}, u, \bar{c}\right)\right]}(\forall)
$$

$f(c, w+1)=0$ iff $A\left(x_{b},(n)_{0}, \bar{c}\right) \rightarrow B\left(z_{b},(n)_{1}, \bar{c}\right)$ is true, and the following condition holds:

$$
\exists k \leq n\left[A\left(x_{b},(\bar{k})_{0}, \bar{c}\right)\right]
$$

Namely

$$
\begin{aligned}
& f(c, w+1)=0 \Leftrightarrow \\
& {\left[A\left(x_{b},(n)_{0}, \bar{c}\right) \rightarrow B\left(z_{b},(n)_{1}, \bar{c}\right)\right] \& \exists k \leq n\left[A\left(x_{b},(\bar{k})_{0}, \bar{c}\right)\right]}
\end{aligned}
$$

Suppose $\sigma(c, w)$ is on an $(\forall)$. Until a witness $k$ such that $A\left(x_{b},(\bar{k})_{0}, \bar{c}\right)$ is found, $f(c, w)=1(w<k)$. After a witness $k$ has been found, $f(c, w)=0(w \geq$ $k)$ as long as $A\left(x_{b},(\bar{n})_{0}, \bar{c}\right) \rightarrow B\left(z_{b},(\bar{n})_{1}, \bar{c}\right)$ is true.

Therefore if the tracing function $\sigma(c, w)$ goes through the upper sequents of the $(\forall)$, then either $\lim _{w \rightarrow \infty} f(c, w)=1$ and $\forall y \neg A\left(x_{b}, y, \bar{c}\right)$, or $\lim _{w \rightarrow \infty} f(c, w)=$ 0 and $\forall u B\left(z_{b}, u, \bar{c}\right)$.

Proposition 7 1. Suppose that $b *\langle n\rangle=\sigma(c, w+1)$ and $\operatorname{Seq}_{c}(b *\langle n\rangle)$ is the $n$-th upper sequent of an inference $(\forall)$. Then $\{f(c, u): \sigma(c, u)=$ $b *\langle m\rangle, m \leq n\}$ changes the values at most twice. Moreover if $f(c, u)=0$ and $f(c, v)=1$ for some $u<v \leq w+1$, then $v=w+1$ and $\sigma(c, v+1)=$ $\sigma(c, v) *\langle 0\rangle$, i.e., $\operatorname{Seq}_{c}(\sigma(c, v))$ is the last upper sequent of the inference $(\forall)$ in the tracing function $\sigma$.

2. (Reduction)

$$
\begin{aligned}
& \text { EA } \vdash \lim _{w \rightarrow \infty} f(c, w)=0 \rightarrow \exists z \forall u B(z, u, c) \\
& \text { EA } \vdash \lim _{w \rightarrow \infty} f(c, w)=1 \rightarrow \exists x \forall y \neg A(x, y, c)
\end{aligned}
$$

Proof. Recall that an inference rule $(\forall)$ in $P_{c}$ is of the form:

$$
\frac{\ldots \quad b *\langle n\rangle: \Gamma, p\left(x_{b *\langle n\rangle}, \bar{n}, \bar{c}\right)=0 \quad \ldots}{b: \Gamma, \forall y\left[p\left(x_{b}, y, \bar{c}\right)=0\right]}(\forall)
$$

where

$$
\left.p\left(x_{b *\langle n\rangle}, \bar{n}, \bar{c}\right)=0 \leftrightarrow\left[A\left(\left((x)_{b *\langle n\rangle}\right)_{0},(\bar{n})_{0}, \bar{c}\right) \rightarrow B\left((x)_{b *\langle k\rangle}\right)_{1},(\bar{n})_{1}, \bar{c}\right)\right]
$$


Let $u$ be such that $\sigma(c, u)=b *\langle m\rangle$ with an $m \leq n$. Then by the definition of the tracing function $\sigma$, we have for $m<n p\left(x_{b *\langle m\rangle}, \bar{m}, \bar{c}\right)=0$, i.e.,

$$
A\left(\left(x_{b *\langle m\rangle}\right)_{0},(\bar{m})_{0}, \bar{c}\right) \rightarrow B\left(\left(x_{b *\langle m\rangle}\right)_{1},(\bar{m})_{1}, \bar{c}\right) .
$$

Suppose there exists a $u \leq w+1$ such that $f(c, u)=0$, and let $u$ denote the minimal such one.

Then for any $v$ with $u \leq v<w+1$, we have $f(c, v)=0$. Therefore if $f(c, v)=1$ for a $v>u$, it must be the case $v=w+1$. This means that for some $k \leq n-1$ with $(k)_{0}=(n)_{0}$

$$
A\left(\left(x_{b}\right)_{0},(k)_{0}, c\right) \wedge \neg B\left(\left(x_{b}\right)_{1},(n)_{1}, c\right) .
$$

Hence $p\left(x_{b}, n, c\right) \neq 0$, and $\sigma(c, v+1)=\sigma(c, v) *\langle 0\rangle$.

Next define $h$ as follows.

1.

$$
h(c, 0)=3 \cdot \operatorname{ord}_{c}(\emptyset) .
$$

In what follows put $a=\sigma(c, w+1)$ and let $\operatorname{Seq}_{c}(a)$ be an upper sequent of an inference $\operatorname{Rule}_{c}(b)$ with $a=b *\langle n\rangle$.

2. The case when $\operatorname{Rule}_{c}(b)$ is an inference rule other than $(\forall)$.

$$
h(c, w+1):=3 \cdot \operatorname{ord}_{c}(\sigma(c, w+1)) .
$$

By Proposition 711 we know that the $f(c, u)$ changes the values at most twice in the upper sequents of an $(\forall)$.

3. The case when $n=0$ and $\operatorname{Rule}_{c}(b)=(\forall)$.

$$
h(c, w+1):=3 \cdot \operatorname{ord}_{c}(\sigma(c, w+1))+2 .
$$

4. The case when $n>0, \operatorname{Rule}_{c}(b)=(\forall)$.

$$
\frac{\cdots \quad b *\langle n\rangle: \Gamma, A\left(x_{b},(\bar{n})_{0}, \bar{c}\right) \rightarrow B\left(z_{b},(\bar{n})_{1}, \bar{c}\right) \quad \cdots}{b: \Gamma, \forall y, u\left[A\left(x_{b}, y, \bar{c}\right) \rightarrow B\left(z_{b}, u, \bar{c}\right)\right]}(\forall)
$$

We have by (5)

$$
\operatorname{ord}_{c}(\sigma(c, w))=\operatorname{ord}_{c}(\sigma(c, w+1)) .
$$

(a) The case when $f(c, w+1)=f(c, w)$.

$$
h(c, w+1):=h(c, w) .
$$

where $\sigma(c, w+1)=\sigma(c, w) \oplus 1$. 
(b) The case when $f(c, w)=1 \& f(c, w+1)=0$.

Then $\sigma(c, w+1)=\sigma(c, w) \oplus 1$ and $n=\min \left\{k: A\left(x_{b},(\bar{k})_{0}, \bar{c}\right)\right\}$.

Let

$$
h(c, w+1):=3 \cdot \operatorname{ord}_{c}(\sigma(c, w+1))+1 .
$$

(c) The case when $f(c, w)=0 \& f(c, w+1)=1$.

This means that $A\left(x_{b},(\bar{n})_{0}, \bar{c}\right) \rightarrow B\left(z_{b},(\bar{n})_{1}, \bar{c}\right)$ is FALSE and $\sigma(c, w+2)=\sigma(c, w+1) *\langle 0\rangle$.

$$
h(c, w+1):=3 \cdot \operatorname{ord}_{c}(\sigma(c, w+1)) .
$$

Obviously $h$ is elementary recursive.

\section{Proposition 8}

$$
\begin{gathered}
\text { (weakly descending) } \mathrm{EA} \vdash 3 K>h(c, w) \geq h(c, w+1) \\
\text { (lowering) EA } \vdash f(c, w) \neq f(c, w+1) \rightarrow h(c, w)>h(c, w+1)
\end{gathered}
$$

Proof. (weakly descending) is obvious.

Consider the case when $\sigma(c, w+1)=a$ and $\operatorname{Seq}_{c}(a)$ is an upper sequent of an inference $\operatorname{Rule}_{c}(b)=(\forall)$ with $a=b *\langle n\rangle$.

If $n=0$, then

$$
h(c, w+1)=3 \cdot \operatorname{ord}_{c}(\sigma(c, w+1))+2<3 \cdot \operatorname{ord}_{c}(\sigma(c, w)) \leq h(c, w)
$$

since $\operatorname{Seq}_{c}(\sigma(c, w))$ is the lower sequent of $\operatorname{Seq}_{c}(a)$ with $b=\sigma(c, w)$.

Assume $n>0$. Using Proposition 7] we see $h(c, w+1) \in\left\{3 \cdot \operatorname{ord}_{c}(\sigma(c, w+\right.$ 1)) $+i: i<3\}$.

Moreover if $\sigma(c, w+2)=\sigma(c, w+1) *\langle 0\rangle$, then

$$
h(c, w+1) \geq 3 \cdot \operatorname{ord}_{c}(\sigma(c, w+1))>3 \cdot \operatorname{ord}_{c}(\sigma(c, w+1))+2 \geq h(c, w+2) .
$$

This completes a proof of Theorems 5 and 4.

\subsection{Provably $\Sigma_{2}^{0}$-functions}

If $\exists z \forall u B(z, u, c)$ is provable for quantifier-free $B$, then we can find a witness $z=\lim _{w \rightarrow \infty} f(c, w)$ as limits of an $f$, whose convergence is ensured by weakly descending chains of ordinals.

Theorem 9 Suppose $I \Sigma_{n}^{0} \vdash \exists z \forall u B(z, u, c)$ for quantifier-free $B$. Then there exist elementary recursive functions $f, h$ and an ordinal $K<\omega_{1+n}$ such that

1.

$$
\text { (weakly descending) EA } \vdash K>h(c, w) \geq h(c, w+1)
$$


2.

$$
\text { (lowering) EA } \vdash f(c, w) \neq f(c, w+1) \rightarrow h(c, w)>h(c, w+1)
$$

3.

$$
\mathrm{EA} \vdash \lim _{w \rightarrow \infty} f(c, w)=z \rightarrow \forall u B(z, u, c)
$$

Proof. As in the proof of Theorem 5, let us define a tracing function $\sigma$.

$\sigma(c, w)$ goes on the leftmost branch up to an $(\forall) . \sigma(c, w)$ goes through the upper sequents of $(\forall)$ as long as side formulas $B\left(z_{a}, \bar{n}, \bar{c}\right)$ is TRUE. If a FALSE side formula $B\left(z_{a}, \bar{n}, \bar{c}\right)$ is found, then throw $z_{a}$ away and go on the leftmost branch.

Now $h$ is defined by $h(c, w):=\operatorname{ord}_{c}(\sigma(c, w))$. $f$ is defined obviously. $f(c, w)=$ $z_{a}$ if $\operatorname{Seq}_{c}(\sigma(c, w))$ is an upper sequent of an $(\forall)$ with its side formula $B\left(z_{a}, \bar{n}, \bar{c}\right)$. Otherwise $f(c, w)$ is arbitrary, say $f(c, w)=0$.

It is well known that the 1-consistency $\operatorname{RFN}_{\Pi_{2}^{0}}\left(I \Sigma_{n}^{0}\right)$ is equivalent over Primitive Recursive Arithmetic PRA to the fact that there is no primitive recursive descending chain of ordinals $<\omega_{1+n}$.

Theorem 10 (Cf. [1] for another form of the 2-consistency of arithmetic.)

The 2-consistency $\operatorname{RFN}_{\Pi_{3}^{0}}\left(I \Sigma_{n}^{0}\right)$ is equivalent over PRA to the fact that every primitive recursive weakly descending chain of ordinals $<\omega_{1+n}$ has a limit, or equivalently to the fact that for any primitive recursive sequence $\{h(c, w)\}_{w}$ of ordinals $<\omega_{1+n}$ the least ordinal $\min _{<_{\varepsilon_{0}}}\{h(c, w): w \in \omega\}$ exists.

\section{Proof.}

Over PRA, $\operatorname{RFN}_{\Pi_{3}^{0}}\left(I \Sigma_{n}^{0}\right)$ yields the existence of the least ordinal $\min _{<_{\varepsilon_{0}}}\{h(c, w)<$ $\left.\omega_{1+n}: w \in \omega\right\}$ since $\alpha=\min _{<_{0}}\{\beta: \exists w[\beta=h(c, w)]\}$ is a $\Sigma_{2}^{0}$-formula.

Conversely let $f(c, w)<2$ be defined as follows:

1. $c$ is not a Gödel number of an $I \Sigma_{n}^{0}$-proof of a $\Sigma_{2}^{0}$-sentence: Then $f(c, w)=$ 0 for any $w$.

2. $c$ is a Gödel number of an $I \Sigma_{n}^{0}$-proof of a $\Sigma_{2}^{0}$-sentence $\exists z \forall u B_{c}(z, u)$ : $f(c, w)$ is defined as in Theorem 9 for a cut free infinite derivation $P_{c}$ of $\exists z \forall u B_{c}(z, u)$. Note that $f$ is non-elementary since it involves cut elimination for predicate logic.

Also let $h(c, w):=\operatorname{ord}_{c}(\sigma(c, w))$.

Then

1.

$$
\text { (weakly descending) PRA } \vdash \omega_{1+n}>h(c, w) \geq h(c, w+1)
$$

2 .

$$
\text { (lowering) PRA } \vdash f(c, w) \neq f(c, w+1) \rightarrow h(c, w)>h(c, w+1)
$$


3.

$$
\mathrm{PRA} \vdash \lim _{w \rightarrow \infty} f(c, w)=0 \rightarrow \operatorname{Prov}_{I \Sigma_{n}^{0}}\left(c,\left\lceil\exists z \forall u B_{c}(z, u)\right\rceil\right) \rightarrow \exists z \forall u B_{c}(z, u)
$$

Therefore

$$
\text { PRA } \vdash \forall c\left[\exists w \forall u \geq w\{h(c, u)=h(c, w)\} \rightarrow \exists \ell\left\{\lim _{w \rightarrow \infty} f(c, w)=\ell\right\}\right]
$$

and

$$
\mathrm{PRA} \vdash \forall c \exists \ell\left[\lim _{w \rightarrow \infty} f(c, w)=\ell\right] \rightarrow \operatorname{RFN}_{\Pi_{3}^{0}}\left(I \Sigma_{n}^{0}\right) .
$$

\section{Provably $\Delta_{2}^{0}$ in EA}

In this section we consider the $\Delta_{2}^{0}$-sets provably in EA.

The following Theorem 11 states that for a set is provably $\Delta_{2}^{0}$ in EA iff it is EA-provably in the class $D_{n}$ of a finite level in the Ershov hierarchy. The finite levels $\left\{D_{n}: n<\omega\right\}$ are called the difference (or Boolean) hierarchy, and by a result due to H. Putnam(Theorem 2 in [7]) we see that a set is provably $\Delta_{2}^{0}$ in EA iff it is equivalent to a Boolean combination of $\Sigma_{1}^{0}$-formulas, provably in EA. This answers to a problem of L. Beklemishev.

Theorem 11 The following are equivalent for quantifier-free $A, B$ and a free variable $c$.

1. EA proves

$$
\forall x \exists y A(x, y, c) \leftrightarrow \exists z \forall u B(z, u, c)
$$

2. There exists a binary elementary recursive predicate $f$, a natural number $K<\omega$ and an elementary recursive function $h: \omega \times \omega \rightarrow K$ such that

(a) (weakly descending)

$$
\mathrm{EA} \vdash K>h(c, w) \geq h(c, w+1)
$$

(b) (lowering)

$$
\mathrm{EA} \vdash f(c, w) \neq f(c, w+1) \rightarrow h(c, w)>h(c, w+1)
$$

(c) (reduction)

$$
\begin{aligned}
& \text { EA } \vdash \lim _{w \rightarrow \infty} f(c, w)=0 \rightarrow \exists z \forall u B(z, u, c) \\
& \text { EA } \vdash \lim _{w \rightarrow \infty} f(c, w)=1 \rightarrow \exists x \forall y \neg A(x, y, c) \\
& \text { EA } \vdash \exists z \forall u B(z, u, c) \rightarrow \forall x \exists y A(x, y, c)
\end{aligned}
$$


for the usual ordering $<$ on $\omega$.

Proof. Assume EA proves (weakly descending) and (lowering) for a natural number $K$. Then EA also proves the convergence of $f$ :

$$
\mathrm{EA} \vdash \exists \ell\left[\lim _{w \rightarrow \infty} f(c, w)=\ell\right],
$$

(reduction) yields

$$
\mathrm{EA} \vdash \forall x \exists y A(x, y, c) \leftrightarrow \lim _{w \rightarrow \infty} f(c, w)=0 .
$$

Conversely suppose that EA proves (44). Then so is the $\exists \forall$-formula

$$
\exists x \exists z \forall y \forall z[A(x, y, c) \rightarrow B(z, u, c)] .
$$

By the Herbrand's theorem there exist a list of variables $\left\{a_{i}, b_{i}: i \leq r\right\}$ and a list of terms $\left\{t_{i}, s_{i}: i \leq r\right\}$ such that

$$
\bigvee\left\{A\left(t_{i}, a_{i}, c\right) \rightarrow B\left(s_{i}, b_{i}, c\right): i \leq r\right\}
$$

is provable in EA, and variables occurring in $t_{i}, s_{i}$ are among $a_{j}, b_{j}$ for $j<i$ besides the parameter $c$.

For simplicity consider the case when $r=1$. Then we have

$$
\mathrm{EA} \vdash \neg A\left(t_{0}, a_{0}, c\right) \vee B\left(s_{0}, b_{0}, c\right) \vee \neg A\left(t_{1}\left(a_{0}, b_{0}\right), a_{1}, c\right) \vee B\left(s_{1}\left(a_{0}, b_{0}\right), b_{1}, c\right)
$$

Let $f$ denote the elementary recursive predicate

$$
f(c, w):= \begin{cases}0 \quad\left[\left\{t_{0} \leq w \wedge \exists y \leq w A\left(t_{0}, y, c\right)\right\} \wedge\left\{s_{0} \leq w \wedge \forall u \leq w B\left(s_{0}, u, c\right)\right\}\right] \vee \\ & {\left[\exists a_{0}, b_{0} \leq w\left\{A\left(t_{0}, a_{0}, c\right) \wedge \neg B\left(s_{0}, b_{0}, c\right) \wedge\right.\right.} \\ & \left.\left.\exists a_{1} \leq w A\left(t_{1}\left(a_{0}, b_{0}\right), a_{1}, c\right) \wedge \forall b_{1} \leq w B\left(s_{1}\left(a_{0}, b_{0}\right), b_{1}, c\right)\right\}\right] \\ 1 \quad \text { otherwise }\end{cases}
$$

For the number

$$
K:=1+2 r+2(=5 \text { if } r=1),
$$

let $h: \omega \times \omega \rightarrow K$ denote the elementary recursive function

$$
h(c, 0)=K-1
$$

and

$$
h(c, w+1):= \begin{cases}h(c, w) & \text { if } f(c, w+1)=f(c, w) \\ h(c, w)-1 & \text { if } f(c, w+1) \neq f(c, w)\end{cases}
$$

Lemma 12 EA proves the facts (weakly descending), (lowering) and (reduction). 
Proof. Argue in EA. (weakly descending) is obvious.

Suppose

$$
\lim _{w \rightarrow \infty} f(c, w)=\ell
$$

for an $\ell=0,1$.

By (4) we have either $\exists z \forall u B(z, u, c)$ or $\exists x \forall y \neg A(x, y, c)$.

First consider the case when $\exists z \forall u B(z, u, c)$. Then $\forall x \exists y A(x, y, c)$. Hence by (77) either $\forall b_{0} B\left(s_{0}, b_{0}, c\right)$ or $\forall b_{1} B\left(s_{1}\left(a_{0}, b_{0}\right), b_{1}, c\right)$ for some $a_{0}, b_{0}$ with $A\left(t_{0}, a_{0}, c\right) \wedge$ $\neg B\left(s_{0}, b_{0}, c\right)$.

If $\forall b_{0} B\left(s_{0}, b_{0}, c\right)$, then $f(c, w)=0$ for any $w \geq \max \left\{t_{0}, s_{0}, y_{0}\right\}$, where $y_{0}=$ $\mu y . A\left(t_{0}, y . c\right)$. Therefore $\ell=0$. Moreover $f(c, w)=1$ for $w<\max \left\{t_{0}, s_{0}, y_{0}\right\}$.

Next assume $\forall b_{1} B\left(s_{1}\left(a_{0}, b_{0}\right), b_{1}, c\right)$ for the minimal $a_{0} . b_{0}$ such that $A\left(t_{0}, a_{0}, c\right) \wedge$ $\neg B\left(s_{0}, b_{0}, c\right)$. Then let $a_{1}$ denote the minimal $a_{1}$ such that $A\left(t_{1}\left(a_{0}, b_{0}\right), a_{1}, c\right)$. We have $f(c, w)=0$ for any $w \geq \max \left\{a_{0}, b_{0}, a_{1}\right\}$, and hence $\ell=0$.

Now consider $w<\max \left\{a_{0}, b_{0}, a_{1}\right\}$. Then $f(c, w)=0$ iff $\max \left\{t_{0}, s_{0}, a_{0}\right\} \leq$ $w<b_{0}$. Therefore $\lambda w . f(c, w)$ changes its values at most three times (when $\left.\max \left\{t_{0}, s_{0}, a_{0}\right\}<b_{0}<a_{1}\right)$.

Next consider the case when $\exists x \forall y \neg A(x, y, c)$. We have $\forall z \exists u \neg B(z, u, c)$. Then $f(c, w)=1$ for any $w$ if $\forall a_{0} \neg A\left(t_{0}, a_{0}, c\right)$, and $f(c, w)=1$ for any $w \geq$ $\max \left\{b_{0}, b_{1}\right\}$ if $\forall a_{1} \neg A\left(t_{1}\left(a_{0}, b_{0}\right), a_{1}, c\right)$ for the minimal $a_{0}, b_{0}, b_{1}$ such that $A\left(t_{0}, a_{0}, c\right) \wedge$ $\neg B\left(s_{0}, b_{0}, c\right)$ and $\neg B\left(s_{1}\left(a_{0}, b_{0}\right), b_{1}, c\right)$. Therefore $\ell=1$.

Finally assume $\forall a_{1} \neg A\left(t_{1}\left(a_{0}, b_{0}\right), a_{1}, c\right)$, and consider $w<\max \left\{b_{0}, b_{1}\right\}$. Then $f(c, w)=0$ iff $\max \left\{t_{0}, s_{0}, a_{0}\right\} \leq w<b_{0}$. Therefore $\lambda w . f(c, w)$ changes its values at most two times in this case.

In any cases, (reduction) was shown, and $\lambda w \cdot f(c, w)$ changes its values at most $1+2 r(=3$ if $r=1)$ times for any $c$, i.e.,

$$
\forall\left(w_{0}<w_{1}<\cdots<w_{1+2 r}\right) \exists i \leq 1+2 r\left[f\left(c, w_{i}\right)=f\left(c, w_{i}+1\right)\right] .
$$

Hence (lowering) follows.

Lemma 12 with a result due to H. Putnam(Theorem 2 in 7]) yields the

Theorem 13 Suppose that EA proves

$$
\forall x \exists y A(x, y, c) \leftrightarrow \exists z \forall u B(z, u, c)
$$

for quantifier-free $A, B$.

Then over $\mathrm{EA} \exists z \forall u B(z, u, c)$ is equivalent to a Boolean combination of $\Sigma_{1}^{0}$ formulas.

Proof. (cf. [7.) Let $r$ be as in (6). Define $\Sigma_{1}^{0} Y_{k}(c)(k \leq 1+2 r)$ and $N_{i}(c)$ by

$$
\begin{aligned}
& Y_{k}(c): \Leftrightarrow \quad \exists\left(w_{0}<w_{1}<\cdots<w_{k-1}\right) \forall i<k\left[f\left(c, w_{i}\right) \neq f\left(c, w_{i}+1\right)\right. \\
&\left.\wedge f\left(c, w_{k-1}\right)=0\right] \\
& N_{k}(c): \Leftrightarrow \quad \exists\left(w_{0}<w_{1}<\cdots<w_{k-1}\right) \forall i<k\left[f\left(c, w_{i}\right) \neq f\left(c, w_{i}+1\right)\right. \\
&\left.\left.\wedge f\left(c, w_{k-1}\right)=1\right]\right]
\end{aligned}
$$


for $k>0$, and $Y_{0}(c): \Leftrightarrow f(c, 0)=0, N_{0}(c): \Leftrightarrow f(c, 0)=1$. Also put $N_{2 r+2}(c): \Leftrightarrow$ $0=1$.

Then EA proves that

$$
\exists z \forall u B(z, u, c) \leftrightarrow \bigvee\left\{Y_{k}(c) \wedge \neg N_{k+1}(c): k \leq 1+2 r\right\}
$$

As in Theorems 9, 10 we see the following theorems.

Theorem 14 Suppose EA $\vdash \exists z \forall u B(z, u, c)$ for quantifier-free B. Then there exist elementary recursive functions $f, h$ and a natural number $K<\omega$ such that

1.

$$
\text { (weakly descending) EA } \vdash K>h(c, w) \geq h(c, w+1)
$$

2.

$$
\text { (lowering) } \mathrm{EA} \vdash f(c, w) \neq f(c, w+1) \rightarrow h(c, w)>h(c, w+1)
$$

3.

$$
\mathrm{EA} \vdash \lim _{w \rightarrow \infty} f(c, w)=z \rightarrow \forall u B(z, u, c)
$$

Theorem 15 The 2-consistency $\mathrm{RFN}_{\Pi_{3}^{0}}(\mathrm{EA})$ is equivalent over PRA to the fact that every primitive recursive weakly descending chain of natural number $<\omega$ has a limit, or equivalently to the fact that for any primitive recursive sequence $\{h(c, w)\}_{w}$ of natural number $<\omega$ the least number $\min _{<}\{h(c, w)<\omega: w \in \omega\}$ exists.

\section{Remark.}

Obviously Theorems 11, 13 and 14 hold for any purely universal extension of $E A$, eg., $E A+C O N(E A)$, PRA.

\section{A Nested limit existence rules}

Every fragment in the Appendix is an extension of Elementary Recursive Arithmetic EA.

In [2, Beklemishev and Visser gave an elegant axiomatization of $\Sigma_{2}^{0}$-consequences of $I \Sigma_{1}^{0}$ in terms of the inference rule $(\operatorname{LimR})$ for limit existence principle:

$$
\frac{\exists m \forall n \geq m h(n+1) \leq h(n)}{\exists m \forall n \geq m h(n)=h(m)}(\operatorname{LimR})
$$

Moreover unnested applications of $(\operatorname{LimR})$ is shown to be equivalent to $I \Pi_{1}^{-}$ (over EA). 
This reminds us another axiomatization of $\Sigma_{2}^{0}$-consequences of $I \Sigma_{1}^{0}$ in [5]. Namely $I \Sigma_{1}^{0}$ is a $\Sigma_{2}^{0}$ conservative extension of $L \Sigma_{1}^{-(\infty)}=\bigcup_{k} L \Sigma_{1}^{-(k)}$, where $L \Sigma_{1}^{-(k)}$ denotes the schema

$$
\begin{aligned}
& \exists x_{1} \ldots \exists x_{k} \theta\left(x_{1}, \ldots, x_{k}\right) \rightarrow \\
& \exists x_{1} \ldots \exists x_{k} \bigwedge_{i=1}^{k}\left[\exists \vec{y} \theta\left(x_{1}, \ldots, x_{i}, \vec{y}\right) \wedge \forall z<x_{i} \forall \vec{y} \neg \theta\left(x_{1}, \ldots, x_{i-1}, z, \vec{y}\right)\right]
\end{aligned}
$$

for $\theta \in \Sigma_{1}^{0}$ without parameters.

For example $L \Sigma_{1}^{-(0)}=\mathrm{EA}$ and $L \Sigma_{1}^{-(1)}=L \Sigma_{1}^{-}=I \Pi_{1}^{-}$.

In this Appendix we show that $L \Sigma_{1}^{-(k)}$ is equivalent to the $k$-nested applications of $(\operatorname{Lim} R)$. To be precise, let $(\operatorname{Lim} R)^{(k)} \vdash$ denote the derivability in the $k$-nested applications of $(\operatorname{LimR}):(\operatorname{LimR})^{(0)} \vdash$ is nothing but EA $\vdash$, and if $(\operatorname{LimR})^{(k)} \vdash \exists m \forall n \geq m h(n+1) \leq h(n)$, then $(\operatorname{LimR})^{(k+1)} \vdash \exists m \forall n \geq$ $m h(n)=h(m)$.

Theorem $16(\operatorname{Lim} R)^{(k)} \vdash \varphi \Leftrightarrow L \Sigma_{1}^{-(k)} \vdash \varphi$ for any $\varphi$.

This is shown by induction on $k$. The proof is obtained by a slight modification of proofs in [2].

First consider

$$
(\operatorname{LimR})^{(k)} \vdash L \Sigma_{1}^{-(k)} .
$$

Let $<^{(k)}(k \geq 1)$ denote the lexicographic order on $k$-tuples of natural numbers. Also $\left\langle x_{1}, \ldots, x_{k}\right\rangle^{(k)}$ denotes a(n elementary recursive) bijective coding of $k$ tuples with its inverses $(n)_{i}^{(k)}(1 \leq i \leq k)$. In what follows the super scripts $(k)$ are omitted.

Then $L \Sigma_{1}^{-(k)}$ says that if there exists an $x$ satisfying $\varphi(x) \equiv \theta\left((x)_{1}, \ldots,(x)_{k}\right)$, then there exists a minimal such $x$ with respect to $<^{(k)}$.

We can assume that EA proves

$$
\exists i\left[\forall j \neq i\left(x_{j}=y_{j}\right) \wedge x_{i}<y_{i}\right] \rightarrow\left\langle x_{1}, \ldots, x_{k}\right\rangle<\left\langle y_{1}, \ldots, y_{k}\right\rangle
$$

Now given a $\Delta_{0}^{0}$-formula $\varphi\left(x_{1}, \ldots, x_{k}, x_{k+1}\right)$ without parameters, we want to show $L \Sigma_{1}^{-(k)}$ with $\theta \equiv \exists x_{k+1} \varphi$.

As in 2] some elementary functions $g_{1}, g, h, h^{\prime}$ are defined successively as follows.

$$
\begin{aligned}
g_{1}(n)= \begin{cases}n & \text { if } \forall y \leq n \neg \varphi\left((y)_{1}, \ldots,(y)_{k},(y)_{k+1}\right) \\
\left\langle(y)_{1}, \ldots,(y)_{k}\right\rangle & \text { otherwise with } y=\mu y \leq n \varphi\left((y)_{1}, \ldots,(y)_{k},(y)_{k+1}\right)\end{cases} \\
g(n)= \begin{cases}\left\langle(n)_{1}, \ldots,(n)_{k}\right\rangle & \text { if } \exists u \leq(n)_{k+1} \varphi\left((n)_{1}, \ldots,(n)_{k}, u\right) \\
g_{1}(n) & \text { otherwise }\end{cases}
\end{aligned}
$$




$$
\begin{aligned}
& h(0)=g(0) \text { and } \\
& h(n+1)= \begin{cases}g(n+1) & \text { if } \forall k, m \leq n(k \neq m \rightarrow g(k) \neq g(m)) \\
g(n+1) & \text { if } \exists m \leq n(g(n+1)=g(m)) \text { and } g(n+1)<<^{(k)} h(n) \\
h(n) & \text { otherwise }\end{cases}
\end{aligned}
$$

Observe that $h(n) \leq \max \{g(m): m \leq n\}$, and hence $h$ is elementary.

$$
h^{\prime}(x)= \begin{cases}h(x) & \text { if } \exists n \leq x \varphi\left((n)_{1}, \ldots,(n)_{k},(n)_{k+1}\right) \\ 0 & \text { otherwise }\end{cases}
$$

Then EA proves that $h^{\prime}$ is eventually decreasing with respect to $<^{(k)}: \exists m \forall n \geq$ $m\left(h^{\prime}(n+1) \leq^{(k)} h^{\prime}(n)\right)$. Therefore $h_{1}^{\prime}(n)=\left(h^{\prime}(n)\right)_{1}$ is eventually decreasing. Hence $\exists y_{1}\left[y_{1}=\lim _{x \rightarrow \infty} h_{1}^{\prime}(x)\right]$ in $(\operatorname{LimR})^{(1)}$.

This in turn implies that $\left\langle\left(h^{\prime}(n)\right)_{2}, \ldots,\left(h^{\prime}(n)\right)_{k}\right\rangle$ is eventually decreasing with respect to $<^{(k-1)}$. Therefore $h_{2}^{\prime}(n)=\left(h^{\prime}(n)\right)_{2}$ is eventually decreasing demonstrably in $(\operatorname{Lim} R)^{(1)}$. Hence $\exists y_{2}\left[y_{2}=\lim _{x \rightarrow \infty} h_{2}^{\prime}(x)\right]$ in $(\operatorname{Lim} R)^{(2)}$, and so on. Therefore $\exists y\left[y=\lim _{x \rightarrow \infty} h^{\prime}(x)\right]$ in $(\operatorname{Lim} R)^{(k)}$.

Now assuming $\exists x_{1} \cdots \exists x_{k} \exists x_{k+1} \theta\left(x_{1}, \ldots, x_{k}, x_{k+1}\right)$, we see as in 2 that $y=$ $\lim _{x \rightarrow \infty} h^{\prime}(x)=\lim _{x \rightarrow \infty} h(x)$, and the limit $y$ is the minimum of $\left\{\left\langle x_{1}, \ldots, x_{k}\right\rangle\right.$ : $\left.\exists x_{k+1} \theta\left(x_{1}, \ldots, x_{k}, x_{k+1}\right)\right\}$ with respect to the lexicographic order $<^{(k)}$ as desired.

Next assume by IH that

$$
L \Sigma_{1}^{-(k)} \vdash \exists m \forall n \geq m h(n+1) \leq h(n) .
$$

We need to show

$$
L \Sigma_{1}^{-(k+1)} \vdash \exists m \forall n \geq m h(n)=h(m) .
$$

For simplicity consider the case $k=1$, and assume that EA proves that

$$
\begin{aligned}
& \left\{\exists x_{1} \varphi_{1}\left(x_{1}\right) \rightarrow \exists x_{1}\left[\varphi_{1}\left(x_{1}\right) \wedge \forall z<x_{1} \neg \varphi_{1}(z)\right]\right\} \wedge \\
& \left\{\exists x_{2} \varphi_{2}\left(x_{2}\right) \rightarrow \exists x_{2}\left[\varphi_{2}\left(x_{2}\right) \wedge \forall z<x_{2} \neg \varphi_{2}(z)\right]\right\} \\
& \rightarrow \exists m \forall n \geq m h(n+1) \leq h(n)
\end{aligned}
$$

Let $\varphi_{i}\left(x_{i}\right) \equiv \exists y \theta_{i}\left(x_{i}, y\right)$.

By the Herbrand's Theorem there exists a sequence of terms $m_{0}\left(a_{1}, a_{2}, b_{1}, b_{2}\right)$, $m_{1}\left(x_{0}, a_{1}, a_{2}, b_{1}, b_{2}\right), m_{2}\left(x_{0}, x_{1}, a_{1}, a_{2}, b_{1}, b_{2}\right), \ldots, m_{k}\left(x_{0}, \ldots, x_{k-1}, a_{1}, a_{2}, b_{1}, b_{2}\right)$ such that the following disjunction is provable in EA:

$$
\begin{aligned}
& \left\{\exists x_{1} \varphi_{1}\left(x_{1}\right) \wedge\left[\neg \theta_{1}\left(a_{1}, b_{1}\right) \vee \exists z<a_{1} \varphi_{1}(z)\right]\right\} \vee \\
& \left\{\exists x_{2} \varphi_{2}\left(x_{2}\right) \wedge\left[\neg \theta_{2}\left(a_{2}, b_{2}\right) \vee \exists z<a_{2} \varphi_{2}(z)\right]\right\} \vee \\
& \left(x_{0} \geq m_{0}\left(a_{1}, a_{2}, b_{1}, b_{2}\right) \rightarrow h\left(x_{0}\right) \geq h\left(x_{0}+1\right)\right) \vee \\
& \left(x_{1} \geq m_{1}\left(x_{0}, a_{1}, a_{2}, b_{1}, b_{2}\right) \rightarrow h\left(x_{1}\right) \geq h\left(x_{1}+1\right)\right) \vee \\
& \ldots \\
& \left(x_{k} \geq m_{k}\left(x_{0}, \ldots, x_{k-1}, a_{1}, a_{2}, b_{1}, b_{2}\right) \rightarrow h\left(x_{k}\right) \geq h\left(x_{k}+1\right)\right)
\end{aligned}
$$


First assume $\exists x_{1} \varphi_{1}\left(x_{1}\right) \wedge \exists x_{2} \varphi_{2}\left(x_{2}\right)$, and pick a minimal $a=\left\langle a_{1}, a_{2}\right\rangle$ such that $\varphi_{1}\left(a_{1}\right) \wedge \varphi_{2}\left(a_{2}\right)$ and $\forall b<a \neg\left[\varphi_{1}\left((b)_{1}\right) \wedge \varphi_{2}\left((b)_{2}\right)\right]$. Then from (8) we see that $\forall z<a_{i} \neg \varphi_{i}(z)$ for $i=1,2$.

Now let

$$
y_{0}=\mu y_{0}\left[\exists b_{1}, b_{2} \exists x_{0} \geq m_{0}\left(a_{1}, a_{2}, b_{1}, b_{2}\right)\left\{\theta_{1}\left(a_{1}, b_{1}\right) \wedge \theta_{2}\left(a_{2}, b_{2}\right) \wedge h\left(x_{0}\right)=y_{0}\right\}\right]
$$

by $L \Sigma_{1}^{-(2)}$.

If $\forall x_{0} \geq m_{0}\left(a_{1}, a_{2}, b_{1}, b_{2}\right)\left(h\left(x_{0}\right) \geq h\left(x_{0}+1\right)\right)$ for some $b_{1}, b_{2}$ with $\theta_{1}\left(a_{1}, b_{1}\right) \wedge$ $\theta_{2}\left(a_{2}, b_{2}\right)$, then $y_{0}=\lim _{x \rightarrow \infty} h(x)$.

Otherwise let

$$
\begin{aligned}
& y_{1}=\mu y_{1}\left[\exists b_{1}, b_{2} \exists x_{0} \geq m_{0}\left(a_{1}, a_{2}, b_{1}, b_{2}\right) \exists x_{1} \geq m_{1}\left(x_{0}, a_{1}, a_{2}, b_{1}, b_{2}\right)\right. \\
& \left.\left(\theta_{1}\left(a_{1}, b_{1}\right) \wedge \theta_{2}\left(a_{2}, b_{2}\right) \wedge h\left(x_{0}\right)<h\left(x_{0}+1\right) \wedge h\left(x_{1}\right)=y_{1}\right)\right]
\end{aligned}
$$

If $\forall x_{1} \geq m_{1}\left(x_{0}, a_{1}, a_{2}, b_{1}, b_{2}\right)\left(h\left(x_{1}\right) \geq h\left(x_{1}+1\right)\right)$ for some $b_{1}, b_{2}$ with $\theta_{1}\left(a_{1}, b_{1}\right) \wedge$ $\theta_{2}\left(a_{2}, b_{2}\right)$, and an $x_{0} \geq m_{0}\left(a_{1}, a_{2}, b_{1}, b_{2}\right)$, then $y_{1}=\lim _{x \rightarrow \infty} h(x)$, and so on.

If $\neg \exists x_{1} \varphi_{1}\left(x_{1}\right)$, then substitute 0 for $a_{1}, b_{1}$ in (9).

\section{References}

[1] T. Arai and G. Mints, Extended normal form theorems for logical proofs from axioms, Theor. Comp. Sci. 232 (2000), 121-132.

[2] L. D. Beklemishev and A. Visser, On the limit existence principles in elementary arithmetic and $\Sigma_{n}^{0}$-consequences of theories, Ann. Pure Appl. Logic 136(2005), 56-74.

[3] Y. l. Ershov, A certain hierarchy of sets. II. Algebra i Logika 7(1968), 15-47.

[4] P. Hájek and P. Pudlák, Metamathematics of First Order Arithmetic, Springer, 1993.

[5] R. Kaye, J. Paris and C. Dimitracopoulos, On parameter free induction schemas, Jour. Symb. Logic 53(1988), 1082-1097.

[6] G.E. Mints, Finite investigations of transfinite derivations, in: Selected Papers in Proof Theory (Bibliopolis, Napoli, 1992) 17-72.

[7] H. Putnam, Trial and error predicates and the solution to a problem of Mostowski, Jour. Symb. Logic 30(1965), 51-57.

[8] H. E. Rose, Subrecursion:Functions and hierarchies. Oxford Logic Guides 9. Oxford University Press 1984.

[9] Th. Skolem, Proof of some theorems on recursively enumerable sets. Notre Dame J. of Formal Logic 3, 65-74 (1962). 
[10] R. Sommer, Transfinite inducton within Peano arithmetic, Ann. Pure Appl. Logic 76 (1995), 231-289.

[11] F. Stephan, Y. Yang and L. Yu, Turing degrees and the Ershov hierarchy, In: T. Arai, J. Brendle, C. T. Chong, R. Downey, Q. Feng, H. Kikyo and H. Ono (eds.), Proceedings of the 10th Asian Logic Conference, 2009, World Scientific, Singapore, pp. 300-321. 